\title{
Rückblick auf 2011 und Ausblick
}

Urs Keller

Korrespondenz:

Dr. med. Urs Keller

FA für Allgemeinmedizin FMH

Büntenstrasse 6

CH-7323 Wangs

urs.keller[at]hin.ch
Am 14. Managed-Care-Zukunfts-Workshop vom 8.10. Dezember 2011 in Scuol trafen sich Vertreter von Managed-Care-Ärztenetzen, Vertreter des Dachverbandes medswiss.net, des Berufsverbandes "Hausärzte Schweiz», vom Forum Managed Care, der Politik und eines Versicherers zum jährlichen Gedankenaustausch.

Zum 14. Managed-Care-(MC)Treffen kamen so viele Teilnehmer wie noch nie. Die 17 Netzvertreter repräsentierten 3700 MC-Ärztinnen und -ärzte, die Vertreter vom Berufsverband Hausärzte Schweiz 5850 Grundversorger und vom MC-Dachverband medswiss.net 2500 MC-Ärzte. Die anwesenden Nationalrätinnen Yvonne Gilli (GP) und Ruth Humbel (CVP) sowie Nationalrat Ignazio Cassis (FDP) unterstrichen mit ihrer Teilnahme die Bedeutung dieser Zusammenkunft.

$\mathrm{Zu}$ Beginn formulierten die Vertreter der MCNetze Perspektiven des zur Debatte stehenden MCGesetzes, das die eidgenössischen Räte beschlossen haben und gegen das das Referendum ergriffen wurde. Mehrheitlich war man der Meinung, dass aktuell kaum die Hälfte der Bevölkerung für dieses Gesetz stimmen würde. Man machte sich Gedanken, warum dies so sei und wie die Bevölkerung und alle medizinischen Leistungserbringer besser über den Inhalt der Vorlage informiert werden könnten. Darüber hinaus wurde über die Weiterentwicklung von MC und mögliche Gefahren bei Ablehnung der Vorlage debattiert. Eine solche hätte wahrscheinlich weitergehende Regulierungen zur Folge, wie z.B. Akzeptanz einer Einheitskasse, Fallen des Kontrahierungszwanges, Versicherer-Netze und eine Verzögerung des verbesserten Risikoausgleichs. Aus der Gesamtheit der Beiträge ging hervor, dass die Argumente für $\mathrm{MC}$ viel schwieriger $\mathrm{zu}$ formulieren und damit auch schwieriger zu vertreten sind als die dagegen, die schlagwortartig bereits für das Referendum verwandt wurden.

Yvonne Gilli, Mitglied eines St. Galler Ärztenetzes und als Nationalrätin (GP) in der Kommission für soziale Sicherheit und Gesundheit SGK aktiv an der MC-Gesetzgebung beteiligt, berichtete, dass vor allem die Visionen aus dem Scuoler Workshop 2008 dem Gesetz Pate standen und praktisch 1:1 umgesetzt wurden. Auch die damals geforderten Eckwerte seien alle mitberücksichtigt worden. Ermessensspielraum auf Verordnungsebene bestehe in den Bereichen Qualitätssicherung, Budgetmitverantwortung, Risikoausgleich und flächendeckender Versorgung. Zentrales Element sei die Einführung der bereits seit langem breit abgestützten Forderung nach Verfeine- rung des Risikoausgleiches durch Berücksichtigung der Morbidität. Es sei auch eine einmalige Chance, eine Gewaltentrennung zwischen Leistungserbringer und Versicherer zu erreichen. Die Gefahr einer gewissen Überregulierung auf Verordnungsebene sei nicht von der Hand zu weisen.

Die Nationalräte Ignazio Cassis (FDP) und Ruth Humbel Näf (CVP) erläuterten aus ihrer Sicht die Vorzüge der Vorlage. Auch sie betonten die Chance, die «Gewaltentrennung» zwischen Versicherern und Leistungserbringern festzuschreiben. Es sei daher absolut nicht verständlich, warum dieses Gesetz mit den Schlagworten «Gesetz der Versicherer» bzw. «alle Macht den Krankenkassen» bekämpft werde.

Aus Sicht der Netze beleuchtete Jörg Fritschi, Präsident des Dachverbandes medswiss.net, die Bedeutung der Gesetzesvorlage und beschrieb den Werdegang der Diskussion über das Referendum während der Ärztekammer und die von ihr bewilligten 2,7 Millionen Franken für das Referendum und die Nein-Kampagne. Kurz wurden auch die noch offenen Punkte der Interpretation von Gesetzesformulierungen und Punkte der Rechtsstaatlichkeitsregeln erläutert. Dies leitete dann über zu einer möglichen Abstimmungskampagne zugunsten der MC-Vorlage.

\section{MC-Vorlagen-Diskussion}

Mit den anwesenden Politikern wurden die Gründe pro und kontra MC-Vorlage nochmals diskutiert. Die Befindlichkeit an der Basis der Netze wurde ausgeleuchtet, und die Gesundheitsbarometer-Resultate wurden diskutiert. Die Gruppe informierte sich, wie eine Abstimmung abläuft und machte sich Gedanken, wie die Netze intern und extern zu gegebener Zeit darüber informiert werden könnten. Dies im Wissen, dass auch eine Minderheit der Netzmitglieder einer solchen Vorlage skeptisch gegenübersteht. Auch das Problem der «Wahrheitsverdrehungen», die als Argumente gegen die Vorlage genutzt werden, wurden diskutiert.

An der Delegiertenversammlung von Hausärzte Schweiz wurde ebenfalls über die Vorlage beraten. Es wurde mit dem Stimmenverhältnis 3:1 beschlossen, dass Hausärzte Schweiz sich aktiv für die MC-Vorlage einsetzen soll. Die dort geäusserten Argumente wurden präsentiert. Auch die Präsentation der Resultate einer Umfrage bei der Kantonalen Ärztegesellschaft des Kantons Zürich (AGZ) wurde heftig diskutiert.

In fünf Arbeitsgruppen wurden Themen zur MCVorlage diskutiert und Thesen erarbeitet, die am folgenden Tag im Plenum finalisiert und in strategische 


\section{Projekte und Präsentationen}

Gruppenpraxen: eastcare-Angebot - Dr. med. R. Buff, eastcare/Präsident vmco MehrFachArzt: erste Erfahrungen mit einem neuen Label - Dr. med. W. Czerwenka, ARGOMED 3 Slogans, 3 Ängste, 3 Werte - Dr. med. M. Enz Kuhn, Hausärzte Schweiz Ärztenetze und Evidence - Dr. med. J. Fritschi, LuMed, Präsident medswiss.net Chronic Care Management in der Praxis - Dr. med. Thomas Grüninger, Centramed Aufnahme von Spezialisten bei medix zürich

Selektiver Aufnahmestopp für spezielle Versicherte (EGK und Assura) -

Dr. med. F. Huber, mediX Zürich

Managed Care: Umfrage bei der AGZ - Dr. med. R. Hurni, Zolamed

Forschung und Schulung in Netzwerken - Dr. med. U. Keller, PizolCare

Polymedikation: Blick in eine Blackbox - Dr. med. C. Marti, mediX Zürich / Medsolution Zürich Rural Networking - was ist anders? - Dr. med. T. Michel, beodocs

Early Feedback Programme - Dr. med. A. Müller, Grisomed

Praxisinformatik - Dr. med. G. Schilling, Hausärzte Schweiz

Chronic Care: Zusammenarbeit Hausärzte und Versicherer - Dr. med. J. Vontobel, Concordia

Neugründung eines Netzes (ZGN) - Dr. med. T. Wettstein, Zürcher Gesundheitsnetz

Online-Praxis - Dr. med. A. Wirthner, mediX Bern

Dezentral vernetzte Landpraxen - Dr. med. F. Weyland, Landpraxen TG les Management von Chronic-Care-Patienten wurden vorgestellt, dazu Aspekte der Arzneimittelsicherheit bei Polymedikation. Ein weiteres Projekt betraf die Verbesserung des Informationsaustausches zwischen Praxis und Spital und informierte über den Nutzen in den Praxen und die Ablehnung bei den Spitalärzten, was dazu führte, dass das Spital diesen Austausch automatisierte und ins Krankenhausinformationssystem (KIS) überführte. Auch die Informationstechnik mit der Praxisinformatik war wieder ein leidiges Thema. Lichtblick ist die Gründung eines unter Führung der Ärzte stehenden Instituts für Praxisinformatik. Dass die Zusammenarbeit im Bereich Chronic Care für eine grosse Krankenversicherung zentral ist, wurde anhand eigener Zahlen aufgezeigt: $10 \%$ der Versicherten generieren 59\% der Kosten und nur $8 \%$ senden keine einzige Rechnung ein.

\section{Hausärzte werden «Mangelware»}

Konzepte umgesetzt wurden. Es gilt, die Argumente für und gegen MC zuerst bei der medizinischen Basis bekannt zu machen und vor allem aufzuzeigen, worum es überhaupt geht, denn viele kennen die Inhalte dieser Vorlage gar nicht. Auch sind die Alternativen und Risiken bei einer Ablehnung, bzw. die Bedeutung dieser Vorlage für die zukünftige Ausgestaltung unseres Gesundheitswesens kaum bekannt.

\section{Zukunft der medizinischen Betreuung in Ärztenetzen}

Die Zukunft der medizinischen Grundversorgung unserer Bevölkerung stand im Vordergrund der Berichte aus den einzelnen Ärztenetzen. Es wurde über Vernetzungsmöglichkeiten in ländlichen Gebieten mit Vor- und Nachteilen berichtet, sowie von der Qualitätskontrolle bzw. dem Nachweis von Qualität in verschiedenen Tools wie «Equam» und «MehrFachArzt». Auch Projekte aus Forschung und Schulung, vor allem im Hinblick auf evidenzbasierte Medizin, wurden präsentiert. Über Neugründungen von Netzen wurde berichtet, die von den Erfahrungen der bestehenden Netze profitieren, aber vor allem neue Visionen in die bestehende Netzlandschaft bringen. Die Integration von Spezialisten scheint nicht ganz so einfach zu sein, einerseits wegen formaler Hindernisse durch die MC-Verträge, andererseits wegen der Anforderungen an das zu übernehmende Gatekeeping. Es zeigten sich deutlich die unterschiedlichen Versorgungsmöglichkeiten von Stadt und Land: In der Stadt herrscht Überfluss an Spezialisten, die interessiert sind an Patientenzuweisungen und mit denen eher selektive Zusammenarbeitsverträge eingegangen werden können. In vorwiegend ländlichen Gebieten, wo meist nur wenig Wahlmöglichkeiten bestehen, arbeiten die Spezialisten bereits eng mit den Grundversorgern zusammen.

Auch neue Versorgungsformen wie die OnlinePraxis, der Betrieb von Gruppenpraxen oder speziel-
Dadurch, dass es für Versicherte zunehmend schwieriger wird, Hausärztinnen und Hausärzte zu finden, wird es für Netze mittelfristig immer attraktiver, sich als Qualitätsgaranten zu positionieren und mit Versicherern, die ihren Versicherten qualitativ gute und kostenoptimierte Leistungen anbieten möchten, attraktive Verträge abzuschliessen.

Ärztenetze müssen aktuell nicht mit allen Kassen kontrahieren. Es wurde vorgeschlagen, einen selektiven Aufnahmestopp für Versicherte von Kassen zu verhängen, mit denen das Netz keinen Vertrag zustande bringt.

\section{Fazit}

An den drei Tagen im winterlichen Scuol wurden weit weg vom Alltag die beiden aktuellen Fragen diskutiert:

- Wie entwickeln sich die Managed-Care-Ärztenetze weiter?

- Wie verhalten sich die Netze angesichts der bevorstehenden Abstimmung über die vom Parlament verabschiedete $\mathrm{MC}$-Vorlage?

Die Teilnahme von drei Nationalräten unterstrich die Bedeutung dieser Themen. Es zeigte sich, dass die Netzentwicklung unverändert dynamisch und typisch schweizerisch unterschiedlich ist. Es gibt unzählige spannende Projekte von Forschung über Onlinepraxis bis Qualitätsnachweis und von Chronic Care bis Versicherungszusammenarbeit. Die politischen Gepflogenheiten wurden diskutiert, und es zeigte sich, dass sich die Netze aktiv für die Annahme des MC-Artikels einsetzen werden.

Zuletzt wurde Ruedi Wartmann gebührend verabschiedet, der nach 40 Jahren Arbeit bei Novartis pensioniert wurde. Er war massgebend verantwortlich bei der Gründung dieses Managed-Care-Workshops vor 14 Jahren und die langjährige Weiterführung dieser Tradition. 